\title{
Drain Amylase Levels in the Early Diagnosis of Gastric Leak after Laparoscopic Sleeve Gastrectomy
}

\author{
Lucia Romano $^{a}$ Antonio Giuliani ${ }^{a}$ Marino Di Furia ${ }^{a}$ Danilo Meloni ${ }^{a}$ \\ Giovanni Cianca $^{a}$ Antonella Mattei $^{b}$ Fabiana Fiasca $^{b}$ Emilio Tonellic \\ Francesco Carlei $^{a}$ Mario Schietroma ${ }^{a}$
}

aDepartment of Biotechnological and Applied Clinical Sciences, Department of General Surgery, University of

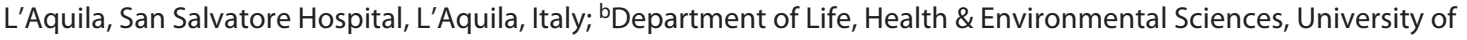
L'Aquila, L'Aquila, Italy; 'U.O.C. di Emergenza e d'Urgenza, S. Salvatore Hospital, L'Aquila, Italy

\section{Highlights of the Study}

- Gastric leak is the most dreaded septic complication after sleeve gastrectomy.

- With the advent of enhanced recovery after surgery (ERAS) protocols, the early detection of gastric leak following laparoscopic sleeve gastrectomy (LSG) is imperative.

- The use of drain amylase levels to detect enteric or pancreatic leak has been described previously.

- In this study, we have investigated the relationship between this index and the appearance of a gastric leak after LSG.

\section{Keywords}

Laparoscopic sleeve gastrectomy · Gastric leak · Amylase

\begin{abstract}
Objectives: Laparoscopic sleeve gastrectomy is gaining popularity as a bariatric option. Gastric leak is the most dreaded septic complication after this procedure. This study investigated levels of drain amylase that could be useful for predicting gastric leak before its clinical presentation. Subjects and Methods: This prospective observational study was carried out in 167 patients who underwent sleeve gastrectomy for morbid obesity between February 2014 and March 2020. Measurement of drain amylase levels (DALs) was adapted as a routine procedure. The results of the receiver operative characteristic (ROC) curve analysis revealed an optimal drain amylase levels cutoff point of $814.18 \mathrm{IU} / \mathrm{L}$.
\end{abstract}

Consequently, the DALs were classified as DALs $<814.18$ or DALs $\geq 814.18$ for all subsequent analyses. Results: Gastric leak occurred in 6 patients. Drain amylase levels of 167 patients were tested. The mean value for patients without leak was $71.13 \pm 72.11 \mathrm{IU} / \mathrm{L}$; for patients with leak, it was 4,687 \pm $6,670 \mathrm{IU} / \mathrm{L}(p<0.001)$. Using ROC curve analysis, the mean \pm standard error of the area under the curve for DALs on postoperative day 1 was $0.9927 \pm 0.0075, \mathrm{Cl}=0.978-1.00$, and a cutoff level at $814.18 \mathrm{IU} / \mathrm{L}$ for predicting gastric leak achieved $83.33 \%$ sensitivity and $100 \%$ specificity with positive predictive value of $100 \%$ and negative predictive value $99.38 \%$. All patients with a leak, but one, had a drain amylase level $>814.18 \mathrm{IU} / \mathrm{L}$. Conclusion: The determination of drain amylase levels after sleeve gastrectomy is a significant indicator of gastric leak with high sensitivity and specificity.

(c) 2021 The Author(s)

Published by S. Karger AG, Basel karger@karger.com www.karger.com/mpp

Karger $\stackrel{\text { ' }}{5}$

BOPEN ACCESS
(C) 2021 The Author(s)

Published by S. Karger AG, Basel

This is an Open Access article licensed under the Creative Commons Attribution-NonCommercial-4.0 International License (CC BY-NC) (http://www.karger.com/Services/OpenAccessLicense), applicable to the online version of the article only. Usage and distribution for commercial purposes requires written permission. 


\section{Introduction}

Laparoscopic sleeve gastrectomy (LSG), first described by Gagner et al. [1], is currently one of the most common surgical bariatric procedures for the treatment of severe obesity $[2,3]$. Among its complications, the most frequent is gastric leak, which has a rate between 0 and $7 \%$ $[4,5]$ and that can lead to abdominal sepsis or chronic gastric fistula $[6,7]$. Other complications, such as hemorrhage $(1.0-1.2 \%)$ and strictures $(0.6-1 \%)$, are less frequent $[8,9]$. With the advent of enhanced recovery after surgery (ERAS) protocols, the early detection of gastric leak following LSG is imperative. Ideally, the methods used to predict leaks should be inexpensive, safe, and easily repeatable. One of the main objectives of current research is to propose an early predictor of this complication.

Amylase is a digestive enzyme found in low concentrations in blood $(<500 \mathrm{IU} / \mathrm{L})$ but high concentration in saliva $(70,000 \mathrm{IU} / \mathrm{L})$. Thus, it is a reasonable assumption that a high concentration of amylase in a juxta-gastric suture drain would represent salivary amylase signifying a gastric leak. The use of drain amylase levels (DALs) to detect enteric or pancreatic leak has been described in multiple previous article $[10,11]$. Moreover, elevated DALs have been associated with leaks in bariatric surgery. Indeed, a high concentration of DALs after Roux-en-Y gastric bypass (RYGB) has been associated with gastrojejunal leak $[12,13]$. However as far as we are aware no studies have investigated the relationship between DALs and the appearance of a gastric leak after LSG. Isil et al. [14] monitored DALs for early diagnosis of gastric leaks in sleeve gastrectomy in a small number of rats. Moreover, amylase levels are different in humans and rats. Phuong et al. [15] is a poster reported the normal range of DALs after LSG in 246 patients. Six patients (2.4\%) have had a postoperative bleeding event, but none of the patients experienced staple line leak. The aim of this prospective observational study was to ascertain whether routine determination of DALs on postoperative day (POD) 1 after LSG could help predict gastric leak before its clinical presentation.

\section{Methods}

We performed a prospective, single center, observational clinical study in 167 consecutive obese patients, who underwent LSG in our center between February 2014 and March 2020. The inclusion criteria were body mass index $>40 \mathrm{~kg} / \mathrm{m}^{2}$ or $35 \mathrm{~kg} / \mathrm{m}^{2}$ with at least 1 obesity-related comorbidity. We considered as contraindi- cations to LSG severe gastroesophageal reflux disease and Barrett's esophagus [16]. Before surgery, patients underwent evaluations by a multidisciplinary team (composed of a psychologist, endocrinologist, gastroenterologist, and nutritionist). The study protocol was approved by the Ethics Committee of the Faculty of the University of L'Aquila, and all patients gave their written informed consent to participate in the study.

\section{Operative Technique}

Our surgical technique has been previously described [4]. All patients were double checked with a methylene blue test and upper gastrointestinal transit (X-ray, computed tomography scan, or endoscopy) on POD2, and if no leakage was detected, a liquid diet was started. The drain was removed on the next day.

Patients were discharged on POD5. We collected a database, including gender, age at the time of surgery, comorbidities, duration of the surgical procedure, eventual conversion from laparoscopic to open procedure, and duration of hospitalization. An intention-to-treat analysis was performed; patients who required conversion to open procedure were included in the analysis. Measurement of drain amylase levels from a silicone suction drain (10Fr Jackson-Pratt drain) placed along the vertical staple line was adopted as a routine procedure. An aliquot of drainage was sent early in the morning of POD1 to the laboratory for amylase determination. The optimal drain amylase levels cutoff values for gastric leak after LSG were calculated using a receiver operative characteristic (ROC) curve analysis. The Youden Index (sensitivity + specificity - 1) was used to select a threshold to estimate sensitivity and specificity. The results of the ROC curve analysis revealed an optimal drain amylase levels cutoff point of 814.18 IU/L. Consequently, the DALs were classified as DALs $<814.18$ or DALs $\geq 814.18$ for all subsequent analysis. Stapling line leak was defined as a leak of contrast material shown by upper gastrointestinal radiography and/or computed tomography scan. Comparison between gastric leak and non-gastric leak groups after LSG was performed. Therefore, the primary endpoint of the study was to evaluate the importance of DALs in predicting gastric leak. The 30-day hospital morbidity and mortality rate were assessed and patients were contacted 30 days after discharge to evaluate complications.

\section{Statistical Analysis}

The characteristics of the study sample were analyzed using descriptive statistics. The discrete and nominal variables were expressed using frequencies and percentages; continuous variables were expressed as means and standard deviations. The Wilcoxon rank-sum test was performed for testing differences between groups, according to the presence or absence of gastric leak. ROC curve analysis was performed, and the respective areas under the curve (AUC) with standard errors and $95 \%$ confidence intervals were calculated to evaluate the predictive value of drain amylase levels on POD1 for the diagnosis of gastric leak as postoperative complication. If the confidence interval did not include the 0.5 value, the parameter had an ability to distinguish between the 2 groups (presence or absence of gastric leak). The sensitivity and specificity of this parameter were calculated. Positive predictive value and negative predictive value were calculated for the specific sensitivity and specificity, considering the disease prevalence. The maximal Youden index (sensitivity + specificity -1 ) was calculated to establish cutoff values. A parameter for which the AUC was equal to 0.05 was not predictive of gastric leak; on the contrary, 
Table 1. Baseline clinical characteristics of the patients $(N=167)$

\begin{tabular}{lccc}
\hline & $\begin{array}{l}\text { Gastric leak } \\
(n=6)\end{array}$ & $\begin{array}{l}\text { No gastric leak } \\
(n=161)\end{array}$ & $p$ value \\
\hline $\begin{array}{lcc}\text { Age, years* } \\
\text { Gender, } n(\%)\end{array}$ & $46 \pm 12$ & $44 \pm 19$ & 0.633 \\
$\quad$ Female & $5(83.33)$ & $102(63.35)$ & 0.317 \\
$\quad$ Male & $1(16.67)$ & $59(36.6)$ & \\
BMI* & $40.05 \pm 2.42$ & $42.15 \pm 4.78$ & 0.242 \\
Comorbidities, $n$ (\%) & & & \\
$\quad$ Type 2 DM & $2(33.33)$ & $40(24.84)$ & 0.637 \\
$\quad$ Hypertension & $3(33.33)$ & $43(26.70)$ & 0.666 \\
$\quad$ Dyslipidemia & $3(50.00)$ & $63(39.13)$ & 0.636 \\
$\quad$ Obstructive sleep apnea & $34.11 \pm 3.24$ & $33.61 \pm 8.20$ & 0.619 \\
Intraoperative blood loss, $\mathrm{mL}^{*}$ & - & $3(1.86)$ & 0.768 \\
Conversion, $n$ (\%) & $60.22 \pm 14.12$ & $58.31 \pm 11.60$ & 0.478 \\
Operative time, min* & $19.61 \pm 10.80$ & $4.52 \pm 1.10$ & $<0.001$ \\
Hospital stay, days* & & & \\
\hline
\end{tabular}

BMI, body mass index; DM, diabetes mellitus. * Mean \pm standard deviation.

Table 2. Mean values of DALs on POD1 between the 2 groups (absence or presence of gastric leak)

\begin{tabular}{lllll}
\hline Parameter & $\begin{array}{l}\text { Total } \\
(N=167)\end{array}$ & Gastric leak & & $p$ value* $^{*}$ \\
\cline { 2 - 4 } & absent $(n=161)$ & present $(n=6)$ & \\
\hline DALs, IU/L, mean \pm SD & $236.97 \pm 1,444.94$ & $71.13 \pm 72.11$ & $4,687.05 \pm 6,670$ & $<0.001$ \\
\hline
\end{tabular}

DALs, drain amylase levels; POD, postoperative day. * Wilcoxon rank-sum test.

a parameter for which the AUC was closest to 1 was the most predictive of gastric leak.

Statistical analyses were carried out using Stata Statistical Software: Release 15 (Stata Corp LP, College Station, TX, USA). All the tests were 2 -tailed, and $p$ values $<0.05$ were considered statistically significant.

\section{Results}

We included in the study 167 patients, of whom 107 (64\%) were females and 60 (35.9\%) were males, with a mean age of $45 \pm 17$ years. Gastric leak was observed in 6 (3.59\%) patients. We noted that the gastric leak rate was related to the learning curve because we diagnosed 4 leaks in the first 50 cases (8\%) and 2 among the cases 51-167 (1.7\%). In 1 patient, leakage was diagnosed on POD5; in 2 patients on POD6; in 2 patients on POD8, and in 1 patient on POD10. Therefore, $83.3 \%$ of the gastric leaks occurred between POD6 and POD10. The patients with gastric leak were endoscopically treated, with a doublepigtail drainage. Further complications (intra-abdominal abscesses) were observed in 2 cases (33.3\%). The abscess was radiologically drained in 1 case, while the other case required laparoscopic surgical toilette and drainage. In these 2 patients, leakage was diagnosed on POD5 and POD6.

The 2 groups of patients (gastric leak and no gastric leak) were compared in terms of age, gender, BMI, comorbidities, intraoperative blood loss and operative time, and no significant differences were reported, whereas the duration of hospitalization was significantly longer in the gastric leak group $(p<0.001)$. These data are showed in Table 1 . One patient $(0.59 \%)$ of the gastric leak group died in hospital on the 30th day because of a massive pulmonary embolism due to sepsis.

The mean values for POD1 DALs in 167 patients were $236.97 \pm 1,444.94 \mathrm{IU} / \mathrm{L}$. The mean values in 161 patients in whom a leak did not develop were $71.13 \pm 72.11 \mathrm{IU} / \mathrm{L}$. The 6 patients with a proved leak had a mean drain amylase of 4,687.05 $\pm 6,670 \mathrm{IU} / \mathrm{L}$. This difference was significant at $p<0.001$ (Table 2).

Using ROC curve analysis, the mean \pm standard error of the area under the curve for DALs on POD1 was 0.9927 
Table 3. Results of ROC curve analysis showing the predictive value of DALs on POD1 for absence/presence of gastric leak after LSG

\begin{tabular}{lllllllll}
\hline Parameter & AUC & 95\% CI & SE & $\begin{array}{l}\text { Cutoff } \\
\text { value }\end{array}$ & $\begin{array}{l}\text { Sensitivity, } \\
\%\end{array}$ & $\begin{array}{l}\text { Specificity, } \\
\%\end{array}$ & $\begin{array}{l}\text { PPV, } \\
\%\end{array}$ & $\begin{array}{l}\text { NPV, } \\
\%\end{array}$ \\
\hline DALs, IU/L & 0.9927 & $0.978-1.00$ & 0.0075 & 814.18 & 83.33 & 100 & 100 & 99.38 \\
\hline
\end{tabular}

ROC, receiver operating characteristic; DALs, drain amylase levels; POD, postoperative day; LSG, laparoscopic sleeve gastrectomy; AUC, area under the curve; CI, confidence interval; SE, standard error; PPV, positive predictive value; NPV, negative predictive value.

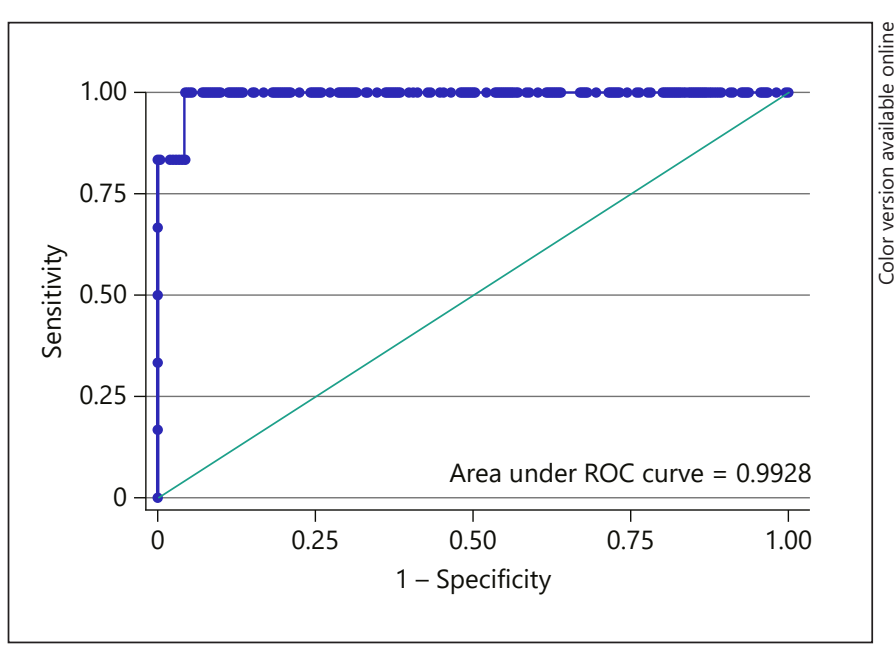

Fig. 1. Receiver operating characteristic (ROC) curve postoperative day 1.

$\pm 0.0075, \mathrm{CI}=0.978-1.00$, and a cutoff level at 814.18 IU/L for predicting gastric leak achieved $83.33 \%$ sensitivity and $100 \%$ specificity with positive predictive value of $100 \%$ and NPV 99.38\% (Table 3; Fig. 1). Only 1 of 6 patients with a gastric leak had drain amylase value $<814.18$ $\mathrm{IU} / \mathrm{L}$, and none of patients without gastric leak had drain amylase value $\geq 814.18 \mathrm{IU} / \mathrm{L}$.

Three patients were readmitted for leak. One was a patient with a normal drain amylase who was readmitted on POD8. This probably represents a delayed leak. The second (POD8) and third (POD10) readmission concerned 2 patients in whom the drain amylase values were elevated, but subsequent contrast study demonstrated no leak. Three patients $(1.79 \%)$ required conversion from laparoscopic to open procedure, 2 out of the first 50 cases $(4 \%)$ of the series, and 1 among cases 51-167 (0.86\%). The conversion occurred because of technical difficulties with fundus dissection due to periesophagitis or prepyloric lesion during greater curva- ture dissection or hemorrhage for lesion of short gastric vessels. In none of these converted cases, gastric leak occurred.

\section{Discussion}

The leakage of the staple line after LSG increases mortality, morbidity, and hospital stay [17]. The reported leak rate is $0-7 \%$ [5]. In our study, this rate was 3.59\% (6/167). The diagnosis of leakage is nowadays still based on clinical signs (pain, fever, and tachycardia), laboratory findings (leukocytosis), methylene blue test, and upper gastrointestinal radiography or CT scan. These diagnostic tools could cause significant delays in diagnosis $[18,19]$, as the early clinical symptoms are very subtle $[18,20]$. Aurora et al. [8] noticed that $50 \%$ of gastric leaks occur after POD10. Also, in our case, $83.3 \%$ of the gastric leaks occurred between POD6 and POD10. Therefore, a useful indicator could help in the early diagnosis of the leakage before the onset of clinical signs.

Many studies have shown that inflammatory scores can improve the accuracy of complication prediction after bariatric surgery [21-24]. The determination of amylase in the drain liquid apparently may be an inexpensive and easily available tool (in the same way as inflammatory markers) to help in this regard. The use of the DALs to detect leaks is based on the presence of abundant salivary amylase in the gastric juice, especially in individuals who undergo gastric resection or gastroplasty. under physiologic conditions, salivary amylase is denatured in the gastric $\mathrm{PH}$, but its levels within the stomach remain high for several hours [25].

There are no validated cutoff values for drain amylase. The cutoff value, adopted by Ribeiro et al. [13] (3 times higher than the serum levels) after RYGB, was based in previously reported studies to detect leaks in upper gastrointestinal and pancreatic surgeries [26, 27]. Maher et 
al. [12] state that "multiple analyses of drain amylase values in patients with and without anastomotic leak, after RYGB, revealed that the best discrimination between the 2 groups occurred when we chose a value of $400 \mathrm{IU} / \mathrm{L}$ as the top of the normal range." Phuong et al. [15] are of the opinion that although they have not experienced gastric leak in their patients after LSG, DALs above $182 \mathrm{IU} / \mathrm{L}$ were statistically outliers (above 1 standard deviation).

In our study, the optimal DAL cutoff was calculated on POD1 using an ROC curve analysis which revealed an optimal DAL cutoff point of $814.18 \mathrm{IU} / \mathrm{L}$. In previous studies, drain amylase monitoring was useful in esophagectomy and pancreaticoduodenectomy [26, 28]. Also, the determination of the DALs after RYGB is a significant indicator of leaks. Maher et al. [12] concluded that DALs are a simple and low-cost adjunct with high sensitivity and specificity that can help identify patients who may have a leak after gastric bypass surgery.

So, though DALs are a reliable early predictor of anastomotic leakage in esophagectomy, pancreaticoduodenectomy and RYGB for sleeve gastrectomy have not yet been studied. Therefore, our work is the first prospective study that evaluates the DALs in patients undergone LSG. The measurement of amylase levels from a drain appears to be sensitive $(83.33 \%)$ and mostly specific $(100 \%)$ in detection of postoperative leak if the amylase level is $\geq 814.14$ IU/L. The sensitivity of $83.33 \%$ in our series may be understated because the 1 patient with a drain amylase $<814.18$ IU/L probably had a delayed anastomotic leak that occurred after discharge.

The study of Maher et al. [12] reported that two-thirds of patients have an elevated DALs ( $>400 \mathrm{IU} / \mathrm{L})$ without a leak. They are of the opinion that in patients without leak, possible sources include pancreatic origin from dissection of the posterior stomach away from the pancreas, spilled saliva at the time of construction of the anastomosis. These issues are not present in LSG. We did not observe any "false positives." Therefore, the measurement of DALs is a simple, quick and inexpensive test to evaluate for gastric suture integrity. When used routinely on POD1, a DAL may identify gastric leak at an early stage in asymptomatic patients, as demonstrated in $83.3 \%$ of patients in our series.

This study, being a preliminary study, had certain limitations. First, it was a single-center study with a limited sample size that restricts the statistical power of our analysis. Second, the amylase assay kit used in this study does not distinguish salivary and pancreatic amylase. Finally, the cutoff values of DALs in different studies may be different because of the cumulative number of patients.
Thus, although these findings are interesting, they need verification in a larger sample. Additional studies at other institutions are needed to evaluate drain amylase values as an accurate and early predictor of gastric leak. Furthermore, there is a need to compare the accuracy of DALs with inflammatory scores in predicting gastric leak. In this regard, preliminary results in our laboratory suggest that the prognostic accuracy of DALs is similar inflammatory markers (C-reactive protein, procalcitonin, fibrinogen, white blood cells, and neutrophil-to-lymphocyte ratio). A nomogram for predicting surgical complications in bariatric surgery patients that incorporates not only clinical indicators [29], but also biochemical markers (DALs, C-reactive protein, procalcitonin, etc.) will further improve predictive accuracy.

\section{Conclusions}

Gastric leak after LSG could be associated with severe complications, that is, intra-abdominal abscess, so it is important to detect the problem as soon as possible to treat it early. Clinical sign, laboratory tests, and radiological examinations could delay the diagnosis. Elevated drain amylase levels at POD1 could help in early detection of gastric leak after LSG. Based on the results of this study, the protocol for our patients without clinical symptoms (no pain, no fever, no tachycardia) is as follows:

1. Patients with normal DALs on POD1 are discharged on POD2 or POD3 without undergoing an upper gastrointestinal transit test.

2. Patients with high values of DALs on POD1 undergo an upper gastrointestinal transit test. If a gastric leak is reported, we manage it with endoscopic placement of a double-pigtail drainage. If no leak is reported, if DALs are still high, another radiographic test is performed in POD5 or POD6. If this control is still negative, the patient is discharged and followed by outpatient checks. Otherwise, if DALs return to normal, the patient is discharged without controls.

We believe that this protocol system with team-based approaches (endoscopist, radiologist, and surgeon) could quickly identify, manage postoperative gastric leak after LSG, and could form the basis of the ERAS protocol.

\section{Statement of Ethics}

The study protocol was approved by the Ethics Committee of the University of L'Aquila, and all patients gave their written informed consent to participate in the study. 


\section{Conflict of Interest Statement}

The authors declare that there is no conflict of interest.

\section{Author Contributions}

All authors contributed to this article.

\section{Funding Sources}

The authors did not receive any funding.

\section{References}

1 Ren CJ, Patterson E, Gagner M. Early results of laparoscopic biliopancreatic diversion with duodenal switch: a case series of 40 consecutive patients. Obes Surg. 2000;10(6):514-24.

2 Cottam D, Qureshi FG, Mattar SG, Sharma S, Holover S, Bonanomi G, et al. Laparoscopic sleeve gastrectomy as an initial weight-loss procedure for high-risk patients with morbid obesity. Surg Endosc. 2006 Jun;20(6):859-63.

3 Carubbi F, Ruscitti P, Pantano I, Alvaro S, Benedetto PD, Liakouli V, et al. Jejunoileal bypass as the main procedure in the onset of immune-related conditions: the model of BADAS. Expert Rev Clin Immunol. 2013 May; 9(5):441-52.

4 Giuliani A, Romano L, Papale E, Puccica I, Di Furia M, Salvatorelli A, et al. Complications of postlaparoscopic sleeve gastric resection: review of surgical technique. Minerva Chir. 2019 Jun;74(3):213-7.

5 Bellanger DE, Greenway FL. Laparoscopic sleeve gastrectomy, 529 cases without a leak: short-term results and technical considerations. Obes Surg. 2011 Feb;21(2):146-50.

6 Giuliani A, Romano L, Marchese M, Necozione S, Cianca G, Schietroma M, et al. Gastric leak after laparoscopic sleeve gastrectomy: management with endoscopic double pigtail drainage. A systematic review. Surg Obes Relat Dis. 2019;15(8):1414-9.

7 Marchese M, Romano L, Giuliani A, Cianca G, Di Sibio A, Carlei F, et al. A case of intrasplenic displacement of an endoscopic doublepigtail stent as a treatment for laparoscopic sleeve gastrectomy leak. Int J Surg Case Rep. 2018;53:367-9.

8 Aurora AR, Khaitan L, Saber AA. Sleeve gastrectomy and the risk of leak: a systematic analysis of 4,888 patients. Surg Endosc. 2012 Jun;26(6):1509-15.

9 Khoursheed M, Al-Bader I, Mouzannar A, Ashraf A, Bahzad Y, Al-Haddad A, et al. Postoperative bleeding and leakage after sleeve gastrectomy: A single-center experience. Obes Surg. 2016 Dec;26(12):3007.

10 Kong J, Gananadha S, Hugh TJ, Samra JS. Pancreatoduodenectomy: role of drain fluid analysis in the management of pancreatic fistula. ANZ J Surg. 2008;78(4):240-4.
11 Morton RP, Mehanna H, Hall FT, McIvor NP. Prediction of pharyngocutaneous fistulas after laryngectomy. Otolaryngol Head Neck Surg. 2007;136(4 Suppl 1):S46-9.

12 Maher JW, Bakhos W, Nahmias N, Wolfe LG, Meador JG, Baugh N, et al. Drain amylase levels are an adjunct in detection of gastrojejunostomy leaks after Roux-en-Y gastric bypass. J Am Coll Surg. 2009;208(5):881-6.

13 Ribeiro IB, Gestic MA, Utrini MP, Chaim FDM, Chaim EA, Cazzo E. Drain amylase levels may indicate gastrojejunostomy leaks after Roux-en-Y gastric bypass. Arq Gastroenterol. 2018 Jan;55(1):66-72.

14 Isil RG, Egin S, Ilhan M, Bademler S, Gokcek B, Yesiltas M, et al. Drain amylase monitoring for early diagnosis of anastomotic leakage in sleeve gastrectomy: an animal study. Ann Ital Chir. 2018;89:562-8.

15 Phuong V, Kuwada ST, Villanueva I, Gersin $\mathrm{K}$, Stefanidis D. What is the normal drain amylase range for laparoscopic sleeve gastrectomy? Presentation at the SAGES 2014. South Lake City, Utah; 2014.

16 Schietroma M, Colozzi S, Romano L, Pessia B, Giuliani A, Vicentini V, et al. Short- and longterm results after laparoscopic floppy Nissen fundoplication in elderly versus non-elderly patients. J Minim Access Surg. 2020 Jul;16(3): 256-63.

17 Sakran N, Goitein D, Raziel A, Keidar A, Beglaibter N, Grinbaum R, et al. Gastric leaks after sleeve gastrectomy: a multicenter experience with 2,834 patients. Surg Endosc. 2013 Jan;27(1):240-5.

18 Albanopoulos K, Alevizos L, Natoudi M, Dardamanis D, Menenakos E, Stamou K, et al. Creactive protein, white blood cells, and neutrophils as early predictors of postoperative complications in patients undergoing laparoscopic sleeve gastrectomy. Surg Endosc. 2013; 27(3):864-71

19 Di Furia M, Romano L, Salvatorelli A, Brandolin D, Lomanto D, Cianca G, et al. Indocyanine green fluorescent angiography during laparoscopic sleeve gastrectomy: preliminary results. Obes Surg. 2019 Dec;29(12): 3786-90.
20 Schietroma M, Pessia B, Mattei A, Romano L Giuliani A, Carlei F. Temperature-neutrophils-multiple organ failure grading for complicated intra-abdominal infections. Surg Infect. 2020;21(1):69-74.

21 Zoubi M, Khidir N, Bashah M. Challenges in the diagnosis of leak after sleeve gastrectomy: clinical presentation, laboratory, and radiological findings. Obes Surg. 2021;31:612-6.

22 Kassir R, Debs T, Petrucciani N, Tiffet O, Amor IB, Gugenheim J. Predictive value of Creactive protein for complications post-laparoscopic Roux-en-Y gastric bypass. Obes Surg. 2017 Sep;27(9):2460-1.

23 Romano L, Mattei A, Colozzi S, Giuliani A, Cianca G, Lazzarin G, et al. Laparoscopic sleeve gastrectomy: a role of inflammatory markers in the early detection of gastric leak. J Minim Access Surg. 2020 Sep 12.

24 Williams M, Langlands F, Giles M. Does Creactive protein day 1 post-surgery have a predictive role for post-operative complications: a single-centre perspective following published meta-analysis. Obes Surg. 2020 Jan; 30(1):347-8.

25 Peyrot Des gachons C, Breslin PA. Salivary amylase: digestion and metabolic syndrome. Curr Diab Rep. 2016;16(10):102.

26 Baker E, HillMK SymanowsiI JSJ, Reames MK, Symanowski J, Hurley SC, Salo JC. Drain amylase aids detection of anastomotic leak after esophagectomy. J Gastrointest Oncol. 2016;7(2):181-8.

27 Schietroma M, Pessia B, Carlei F, Amicucci G. Intestinal permeability changes, systemic endotoxemia, inflammatory serum markers and sepsis after Whipple's operation for carcinoma of the pancreas head. Pancreatology. 2017 Sep;17(5):839-46.

28 Soreide K, Labori KJ. Risk factors and preventive strategies for post-operative pancreatic fistula after pancreatic surgery: a comprehensive review. Scand J Gastroenterol. 2016; 51(10):1147-54.

29 Turner PL, Saager L, Dalton J, Abd-Elsayed A, Roberman D, Melara P, et al. A nomogram for predicting surgical complications in bariatric surgery patients. Obes Surg. 2011;21(5):65562. 